The Journal of $\mathbf{N}_{\text {onlinear }} \mathbf{S}_{\text {cience and }}$ Applications

http://www.tjnsa.com

\title{
PROOFS OF THREE OPEN INEQUALITIES WITH POWER-EXPONENTIAL FUNCTIONS
}

\author{
VASILE CIRTOAJE \\ Dedicated to Themistocles M. Rassias on the occasion of his sixtieth birthday \\ Communicated by Choonkil Park
}

\begin{abstract}
The main aim of this paper is to give a complete proof to the open inequality with power-exponential functions

$$
a^{e a}+b^{e b} \geq a^{e b}+b^{e a}
$$

which holds for all positive real numbers $a$ and $b$. Notice that this inequality was proved in [1] for only $a \geq b \geq \frac{1}{e}$ and $\frac{1}{e} \geq a \geq b$. In addition, other two open inequalities with power-exponential functions are proved, and three new conjectures are presented.
\end{abstract}

\section{INTRODUCTION}

We conjectured in [1] and [3] that $e$ is the greatest possible value of a positive real number $r$ such that the following inequality holds for all positive real numbers $a$ and $b$ :

$$
a^{r a}+b^{r b} \geq a^{r b}+b^{r a} .
$$

In addition, we proved in [1] the following results related to this inequality.

Theorem A. If (1.1) holds for $r=r_{0}>0$, then it holds for all $0<r \leq r_{0}$.

Theorem B. If $\max \{a, b\} \geq 1$, then (1.1) holds for any $r>0$.

Theorem C. If $r>e$, then (1.1) does not hold for all positive real numbers a and $b$.

Date: Received: August 19, 2010; Revised: November 24, 2010.

(C) 2011 N.A.G.

2000 Mathematics Subject Classification. Primary 26D10.

Key words and phrases. Open inequality; Power-exponential function; AM-GM inequality. 
Theorem D. If $a$ and $b$ are positive real numbers such that either $a \geq b \geq \frac{1}{r}$ or $\frac{1}{r} \geq a \geq b$, then (1.1) holds for all $0<r \leq e$.

\section{MAin ReSUlT}

In order to give a complete answer to our problem, we only need to prove the following theorem.

Theorem 2.1. If $a$ and $b$ are positive real numbers such that $0<b \leq \frac{1}{e} \leq a \leq 1$, then

$$
a^{e a}+b^{e b} \geq a^{e b}+b^{e a} .
$$

The proof of Theorem 2.1 relies on the following four lemmas.

Lemma 2.1. If $x>0$, then

$$
x^{x}-1 \geq(x-1) e^{x-1} .
$$

Lemma 2.2. If $0<y \leq 1$, then

$$
1-\ln y \geq e^{1-y} .
$$

Lemma 2.3. If $x \geq 1$, then

$$
\ln x \geq(x-1) e^{1-x} .
$$

Lemma 2.4. If $x \geq 1$ and $0<y \leq 1$, then

$$
x^{y-1} \geq y^{x-1} .
$$

Notice that Lemma 2.1 is a particular case of Theorem 2.1, namely the case where $a=\frac{x}{e}$ and $b=\frac{1}{e}$.

On the other hand, from Theorem $\mathrm{B}$ and its proof in [1], it follows that $a, b \in$ $(0,1]$ is the main case of the inequality $(1.1)$. However, we conjecture that the following sharper inequality still holds in the same conditions:

Conjecture 2.1. If $a, b \in(0,1]$ and $r \in(0, e]$, then

$$
2 \sqrt{a^{r a} b^{r b}} \geq a^{r b}+b^{r a} \text {. }
$$

In the particular case $r=2$, we get the elegant inequality

$$
2 a^{a} b^{b} \geq a^{2 b}+b^{2 a}
$$

which is also an open problem. A similar inequality is

$$
2 a^{a} b^{b} \geq(a b)^{a}+(a b)^{b}
$$

where $a, b \in(0,1]$. Notice that a proof of (2.2) is given in [2]. It seems that this inequality can be extended to three variables, as follows.

Conjecture 2.2. If $a, b, c \in(0,1]$, then

$$
3 a^{a} b^{b} c^{c} \geq(a b c)^{a}+(a b c)^{b}+(a b c)^{c} .
$$




\section{PRoOf OF LEMMAS}

Proof of Lemma 2.1. Write the desired inequality as $f(x) \geq 0$, where

$$
f(x)=x \ln x-\ln \left[1+(x-1) e^{x-1}\right]
$$

has the derivatives

$$
f^{\prime}(x)=1+\ln x-\frac{x e^{x-1}}{1+(x-1) e^{x-1}}
$$

and

$$
f^{\prime \prime}(x)=\frac{x(x-1) e^{x-1}\left(e^{x-1}-1\right)+\left(e^{x-1}-1\right)^{2}}{x\left[1+(x-1) e^{x-1}\right]^{2}} .
$$

Since $(x-1)\left(e^{x-1}-1\right) \geq 0$, we have $f^{\prime \prime}(x) \geq 0$, and hence $f^{\prime}(x)$ is strictly increasing for $x>0$. Since $f^{\prime}(1)=0$, it follows that $f^{\prime}(x)<0$ for $0<x<1$, and $f^{\prime}(x)>0$ for $x>1$. Therefore, $f(x)$ is strictly decreasing on $(0,1]$ and strictly increasing on $[1, \infty)$, and then $f(x) \geq f(1)=0$.

Proof of Lemma 2.2. We need to show that $f(y) \geq 0$ for $0<y \leq 1$, where

$$
f(y)=1-\ln y-e^{1-y} .
$$

Write the derivative in the form

$$
f^{\prime}(y)=\frac{e^{1-y} g(y)}{y}
$$

where

$$
g(y)=y-e^{y-1} .
$$

Since $g^{\prime}(y)=1-e^{y-1}>0$ for $0<y<1, g(y)$ is strictly increasing, $g(y) \leq g(1)=$ $0, f^{\prime}(y)<0$ for $0<y<1, f(y)$ is strictly decreasing, and hence $f(y) \geq f(1)=$ 0 .

Proof of Lemma 2.3. Since

$$
e^{1-x}=\frac{1}{e^{x-1}} \leq \frac{1}{1+(x-1)}=\frac{1}{x},
$$

it suffices to show that $f(x) \geq 0$ for $x \geq 1$, where

$$
f(x)=\ln x+\frac{1}{x}-1 .
$$

This is true because $f^{\prime}(x)=\frac{x-1}{x^{2}} \geq 0, f(x)$ is strictly increasing, and hence $f(x) \geq f(1)=0$.

Proof of Lemma 2.4. Consider the nontrivial case when $0<y<1$. For fixed $y \in(0,1)$, we write the desired inequality as $f(x) \geq 0$ for $x \geq 1$, where

$$
f(x)=(y-1) \ln x-(x-1) \ln y .
$$

We have

$$
f^{\prime}(x)=\frac{y-1}{x}-\ln y \geq y-1-\ln y .
$$


Let us denote $g(y)=y-1-\ln y$. Since $g^{\prime}(y)=1-\frac{1}{y}<0, g(y)$ is strictly decreasing on $(0,1)$, and then $g(y)>g(1)=0$. Therefore, $f^{\prime}(x)>0, f(x)$ is strictly increasing for $x \geq 1$, and hence $f(x) \geq f(1)=0$.

\section{Proof of Theorem 2.1}

Making the substitutions $x=e a$ and $y=e b$, we have to show that

$$
\left(x^{x}-y^{x}\right) e^{-x}+\left(y^{y}-x^{y}\right) e^{-y} \geq 0
$$

for $0<y \leq 1 \leq x \leq e$. By Lemma 2.1, we have

$$
x^{x} \geq 1+(x-1) e^{x-1}
$$

and

$$
y^{y} \geq 1+(y-1) e^{y-1} .
$$

Therefore, it suffices to show that

$$
\left(1+(x-1) e^{x-1}-y^{x}\right) e^{-x}+\left(1+(y-1) e^{y-1}-x^{y}\right) e^{-y} \geq 0,
$$

which is equivalent to

$$
x+y-2+\left(1-y^{x}\right) e^{1-x}+\left(1-x^{y}\right) e^{1-y} \geq 0 .
$$

For fixed $y \in(0,1]$, write this inequality as $f(x) \geq 0$, where

$$
f(x)=x+y-2+\left(1-y^{x}\right) e^{1-x}+\left(1-x^{y}\right) e^{1-y}, \quad 1 \leq x \leq e .
$$

If $f^{\prime}(x) \geq 0$, then $f(x) \geq f(1)=0$, and the conclusion follows. We have

$$
f^{\prime}(x)=1-e^{1-x}-y x^{y-1} e^{1-y}+y^{x}(1-\ln y) e^{1-x}
$$

and, by Lemma 2.2 , it follows that

$$
f^{\prime}(x) \geq 1-e^{1-x}-y x^{y-1} e^{1-y}+y^{x} e^{2-x-y} .
$$

For fixed $x \in[1, e]$, let us denote

$$
g(y)=1-e^{1-x}-y x^{y-1} e^{1-y}+y^{x} e^{2-x-y}, \quad 0<y \leq 1 .
$$

We need to show that $g(y) \geq 0$. Since $g(1)=0$, it suffices to prove that $g^{\prime}(y) \leq 0$ for $0<y \leq 1$. We have

$$
e^{y-1} g^{\prime}(y)=(y-1) x^{y-1}-y x^{y-1} \ln x+\left(x y^{x-1}-y^{x}\right) e^{1-x}
$$

and, by Lemma 2.3, we get

$$
e^{y-1} g^{\prime}(y) \leq(y-1) x^{y-1}+\left(y x^{y-1}-y x^{y}+x y^{x-1}-y^{x}\right) e^{1-x} .
$$

If $y x^{y-1}-y x^{y}+x y^{x-1}-y^{x} \leq 0$, then clearly $g^{\prime}(y) \leq 0$. Consider now that $y x^{y-1}-y x^{y}+x y^{x-1}-y^{x}>0$. Since $e^{1-x} \leq \frac{1}{x}$, we have

$$
\begin{aligned}
e^{y-1} g^{\prime}(y) & \leq(y-1) x^{y-1}+\frac{y x^{y-1}-y x^{y}+x y^{x-1}-y^{x}}{x} \\
& =\frac{(x-y)\left(y^{x-1}-x^{y-1}\right)}{x},
\end{aligned}
$$

and, by Lemma 2.4, it follows that $g^{\prime}(y) \leq 0$. Thus, the proof is completed. 


\section{Other Related inequalities}

We posted in [1] the following two open inequalities.

Proposition 5.1. If $a, b$ are nonnegative real numbers satisfying $a+b=2$, then

$$
a^{3 b}+b^{3 a} \leq 2
$$

with equality for $a=b=1$.

Proposition 5.2. If $a, b$ are nonnegative real numbers satisfying $a+b=1$, then

$$
a^{2 b}+b^{2 a} \leq 1
$$

with equality for $a=b=\frac{1}{2}$, for $a=0$ and $b=1$, and for $a=1$ and $b=0$.

A complicated solution of Proposition 5.1 was given by L. Matejicka in [4]. We will give further a much simpler proof of Proposition 5.1, and a proof of Proposition 5.2. However, it seems that the following generalization of Proposition 5.2 holds.

Conjecture 5.1. Let $a, b$ be nonnegative real numbers satisfying $a+b=1$. If $k \geq 1$, then

$$
a^{(2 b)^{k}}+b^{(2 a)^{k}} \leq 1
$$

\section{Proof of Proposition 5.1}

Without loss of generality, assume that $a \geq b$. For $a=2$ and $b=0$, the desired inequality is obvious. Otherwise, using the substitutions $a=1+x$ and $b=1-x$, $0 \leq x<1$, we can write the inequality as

$$
e^{3(1-x) \ln (1+x)}+e^{3(1+x) \ln (1-x)} \leq 2 .
$$

Applying Lemma 6.1 below, it suffices to show that $f(x) \leq 2$, where

$$
f(x)=e^{3(1-x)\left(x-\frac{x^{2}}{2}+\frac{x^{3}}{3}\right)}+e^{-3(1+x)\left(x+\frac{x^{2}}{2}+\frac{x^{3}}{3}\right)} .
$$

If $f^{\prime}(x) \leq 0$ for $x \in[0,1)$, then $f(x)$ is decreasing, and hence $f(x) \leq f(0)=2$. Since

$$
\begin{aligned}
f^{\prime}(x) & =\left(3-9 x+\frac{15}{2} x^{2}-4 x^{3}\right) e^{3 x-\frac{9 x^{2}}{2}+\frac{5 x^{3}}{2}-x^{4}} \\
& -\left(3+9 x+\frac{15}{2} x^{2}+4 x^{3}\right) e^{-3 x-\frac{9 x^{2}}{2}-\frac{5 x^{3}}{2}-x^{4}},
\end{aligned}
$$

$f^{\prime}(x) \leq 0$ is equivalent to

$$
e^{-6 x-5 x^{3}} \geq \frac{6-18 x+15 x^{2}-8 x^{3}}{6+18 x+15 x^{2}+8 x^{3}} .
$$

For the nontrivial case $6-18 x+15 x^{2}-8 x^{3}>0$, we rewrite the required inequality as $g(x) \geq 0$, where

$$
g(x)=-6 x-5 x^{3}-\ln \left(6-18 x+15 x^{2}-8 x^{3}\right)+\ln \left(6+18 x+15 x^{2}+8 x^{3}\right) .
$$


If $g^{\prime}(x) \geq 0$ for $x \in[0,1)$, then $g(x)$ is increasing, and hence $g(x) \geq g(0)=0$. From

$$
\frac{1}{3} g^{\prime}(x)=-2-5 x^{2}+\frac{\left(6+8 x^{2}\right)-10 x}{6+15 x^{2}-\left(18 x+8 x^{3}\right)}+\frac{\left(6+8 x^{2}\right)+10 x}{6+15 x^{2}+\left(18 x+8 x^{3}\right)},
$$

it follows that $g^{\prime}(x) \geq 0$ is equivalent to

$$
2\left(6+8 x^{2}\right)\left(6+15 x^{2}\right)-20 x\left(18 x+8 x^{3}\right) \geq\left(2+5 x^{2}\right)\left[\left(6+15 x^{2}\right)^{2}-\left(18 x+8 x^{3}\right)^{2}\right] .
$$

Since

$$
\left(6+15 x^{2}\right)^{2}-\left(18 x+8 x^{3}\right)^{2} \leq\left(6+15 x^{2}\right)^{2}-324 x^{2}-288 x^{4} \leq 4\left(9-36 x^{2}\right),
$$

it suffices to show that

$$
\left(3+4 x^{2}\right)\left(6+15 x^{2}\right)-5 x\left(18 x+8 x^{3}\right) \geq\left(2+5 x^{2}\right)\left(9-36 x^{2}\right) .
$$

This reduces to $6 x^{2}+200 x^{4} \geq 0$, which is clearly true.

Lemma 6.1. If $t>-1$, then

$$
\ln (1+t) \leq t-\frac{t^{2}}{2}+\frac{t^{3}}{3} .
$$

Proof. We need to prove that $f(t) \geq 0$, where

$$
f(t)=t-\frac{t^{2}}{2}+\frac{t^{3}}{3}-\ln (1+t)
$$

Since

$$
f^{\prime}(t)=\frac{t^{3}}{t+1},
$$

$f(t)$ is decreasing on $(-1,0]$ and increasing on $[0, \infty)$. Therefore, $f(t) \geq f(0)=$ 0 .

\section{Proof of Proposition 5.2}

Without loss of generality, assume that

$$
0 \leq b \leq \frac{1}{2} \leq a \leq 1 .
$$

Applying Lemma 7.1 below for $c=2 b, 0 \leq c \leq 1$, we get

$$
a^{2 b} \leq(1-2 b)^{2}+4 a b(1-b)-2 a b(1-2 b) \ln a,
$$

which is equivalent to

$$
a^{2 b} \leq 1-4 a b^{2}-2 a b(a-b) \ln a .
$$

Similarly, applying Lemma 7.2 for $d=2 a-1, d \geq 0$, we get

$$
b^{2 a-1} \leq 4 a(1-a)+2 a(2 a-1) \ln (2 a+b-1),
$$

which is equivalent to

$$
b^{2 a} \leq 4 a b^{2}+2 a b(a-b) \ln a .
$$

Adding up (7.1) and (7.2), the desired inequality follows. 
Lemma 7.1. If $0<a \leq 1$ and $c \geq 0$, then

$$
a^{c} \leq(1-c)^{2}+a c(2-c)-a c(1-c) \ln a,
$$

with equality for $a=1$, for $c=0$, and for $c=1$.

Proof. Using the substitution $a=e^{-x}, x \geq 0$, we need to prove that $f(x) \geq 0$, where

$$
\begin{gathered}
f(x)=(1-c)^{2} e^{x}+c(2-c)+c(1-c) x-e^{(1-c) x}, \\
f^{\prime}(x)=(1-c)\left[(1-c) e^{x}+c-e^{(1-c) x}\right] .
\end{gathered}
$$

If $f^{\prime}(x) \geq 0$ for $x \geq 0$, then $f(x)$ is increasing, and $f(x) \geq f(0)=0$. In order to prove this, we consider two cases. For $0 \leq c \leq 1$, by the weighted AM-GM inequality, we have

$$
(1-c) e^{x}+c \geq e^{(1-c) x},
$$

and hence $f^{\prime}(x) \geq 0$. For $c \geq 1$, by the weighted AM-GM inequality, we have

$$
(c-1) e^{x}+e^{(1-c) x} \geq c,
$$

and hence $f^{\prime}(x) \geq 0$, too.

Lemma 7.2. If $0 \leq b \leq 1$ and $d \geq 0$, then

$$
b^{d} \leq 1-d^{2}+d(1+d) \ln (b+d),
$$

with equality for $d=0$, and for $b=0, d=1$.

Proof. Excepting the equality cases, from

$$
1-d+d \ln (b+d) \geq 1-d+d \ln d \geq 0,
$$

we get $1-d+d \ln (b+d)>0$. So, we may write the required inequality as

$$
\ln (1+d)+\ln [1-d+d \ln (b+d)] \geq d \ln b .
$$

Using the substitution $b=e^{-x}-d,-\ln (1+d) \leq x \leq-\ln d$, we need to prove that $f(x) \geq 0$, where

$$
f(x)=\ln (1+d)+\ln (1-d-d x)+d x-d \ln \left(1-d e^{x}\right) .
$$

Since

$$
f^{\prime}(x)=\frac{d^{2}\left(e^{x}-1-x\right)}{(1-d-d x)\left(1-d e^{x}\right)} \geq 0,
$$

$f(x)$ is increasing, and hence

$$
f(x) \geq f(-\ln (1+d))=\ln \left[1-d^{2}+d(1+d) \ln (1+d)\right] .
$$

To complete the proof, we only need to show that $-d^{2}+d(1+d) \ln (1+d) \geq 0$; that is,

$$
(1+d) \ln (1+d) \geq d
$$

This inequality follows from $e^{x} \geq 1+x$ for $x=\frac{-d}{1+d}$. 


\section{REFERENCES}

1. V. Cirtoaje, On some inequalities with power-exponential functions, J. Ineq. Pure Appl. Math., 10 (1) (2009), Art. 21, [http://www.emis.de/journals/JIPAM/article 1077.html?sid=1077]. (document), 1, 1, 2, 5

2. V. Cirtoaje, Art of Problem Solving Forum, Jan. 2010, [http://www.artofproblemsolving. com/Forum/viewtopic.php?t=327116]. 2

3. V. Cirtoaje and A. Zeikii, Art of Problem Solving Forum, Nov. 2006, [http:// www.artofproblemsolving.com/Forum/viewtopic.php?t=118722]. 1

4. L. Matejicka, Solution of one conjecture on inequalities with power-exponential functions, J. Ineq. Pure Appl. Math., 10 (3) (2009), Art. 72, [http://www. emis.de/journals/JIPAM/article1128.html?sid=1128]. 5

* Department of Automatic Control and Computers, University of Ploiesti, ROMANIA.

E-mail address: vcirtoaje@upg-ploiesti.ro 